\title{
THE BRAUER GROUP OF A CLOSED CATEGORY
}

\section{J. FISHER-PALMQUIST}

ABSTRACT. The Brauer group of a finitely bicomplete closed category is defined. This group gives known Brauer groups for the appropriate choices of the closed category. There is a Brauer group functor from the category of commutative monoids in the closed category to the category of Abelian groups.

For a finitely bicomplete closed category $\mathbf{V}[8, \mathrm{p} .180]$ we define the Brauer group of $\mathbf{V}, B(\mathbf{V})$. This paper depends heavily on the calculus of Morita contexts given in [7]. If $\mathbf{V}$ is the category of left-modules over a commutative ring $R$, we have $B(\mathbf{V})$ is the Brauer group of $R$ as defined in [2]; if $\mathbf{V}$ is the category of sheaves of modules over a commutative ringed space $(X, \mathcal{U})$, we have $B(V)$ is the Brauer group of $(X, \mathcal{U})$ as defined in [1]; if $\mathbf{V}$ is the category of $Z / 2 Z$ graded $R$-modules $M$ with $R$ a commutative ring concentrated in degree zero and with symmetric homomorphism $c: M \otimes M^{\prime} \rightarrow M^{\prime} \otimes M$ defined by

$$
c\left(m \otimes m^{\prime}\right)=(-1)^{\delta m \cdot \delta m^{\prime}}\left(m^{\prime} \otimes m\right)
$$

for homogeneous elements $m \in M$ and $m^{\prime} \in M^{\prime}$ of respective degrees $\delta m$ and $\delta m^{\prime}$, then $B(\mathbf{V})$ is the Brauer-Wall group of $R$ as defined in [4]. We give necessary conditions on a functor $T$ from one closed category $\mathbf{V}$ to another $\mathbf{V}^{\prime}$ to insure that $T$ induces a homomorphism from $B(\mathbf{V})$ to $B\left(\mathbf{V}^{\prime}\right)$. As a corollary we see that $B$ induces a functor from the category of commutative monoids of $\mathbf{V}[8, \mathrm{p} .167]$ to the category of Abelian groups.

We recall that there is a one-to-one correspondence between monoids $\Lambda$ in $\mathbf{V}$ and one-object $\mathbf{V}$-categories [ $\Lambda$ ]. The opposite monoid $\Lambda^{\circ}$ corresponds to the $V$-category $[\Lambda]^{\circ}$ and the enveloping monoid $\Lambda^{\mathrm{e}}$ corresponds to the $V$-category $[\Lambda] \otimes[\Lambda]^{\circ}$. We will denote the functor category $V^{[\Lambda]}$ by

Presented to the Society, January 25, 1973 under the title Morita contexts and the Brauer group of a closed category; received by the editors July 20, 1973 and, in revised form, February 26, 1974.

AMS (MOS) subject classifications (1970). Primary 18D15, 18D35; Secondary 13 A20, 16 A16.

Key words and phrases. Closed category, Brauer group, monoid, atom, dense, equivalence of categories, monoidal category. 
$\mathbf{V}^{\Lambda}$. A monoid $\Lambda$ is said to be commutative if the identity map from $\Lambda$ to $\Lambda^{\circ}$ is a map of monoids.

For any $\mathbf{V}$-category $\mathbf{A}$ the center of $\mathbf{A}, Z(\mathbf{A})$, is defined by

$$
Z(\mathbf{A})=\int_{A} \mathbf{A}(A, A)
$$

i.e., the end of the $\mathbf{V}$-functor $\mathbf{A}(-,-): \mathbf{A}^{\circ} \otimes \mathbf{A} \rightarrow \mathbf{V}$, if it exists [6, (1.6)]. As an example $Z(V)=I$. We note that if $V_{0}: V^{\circ} \otimes V \rightarrow$ Sets is the underlying hom functor of $\mathbf{V}$ then $\mathbf{V}_{0}(I, Z(A))$ is the set of natural transformations from the identity functor of $A$ to itself. This set is called the center of the set based category $A_{0}$ by Bass [4, p. 55]. If $\mathbf{A}$ is small then $Z(A)$ always exists. If $Z(A)$ exists then it has the unique structure of a commutative monoid such that the projection maps $p_{A}: Z(\mathbf{A}) \rightarrow \mathbf{A}(A, A)$ are maps of monoids for every object $A$ in A.

Proposition 1. Let $\mathbf{C}$ be a small V-category where $\mathbf{V}$ is a bicomplete closed category. Then

(i) $Z\left(\mathbf{V}^{\mathbf{C}}\right)$ exists and $Z\left(\mathbf{V}^{\mathbf{C}}\right)=Z(\mathbf{C})$;

(ii) $Z(\mathbf{C})=V^{C \otimes C^{0}}(\mathbf{C}(-,-), C(-,-))$.

Proof. This follows from the V-Yoneda isomorphism [6, (1.19)] and the universal properties of ends.

If $\Lambda$ is a monoid then its center, $Z(\Lambda)$, is defined to be $Z([\Lambda])$, which by Proposition 1 (ii) is $V^{\Lambda^{e}}(\Lambda, \Lambda)$. Call $\Lambda$ central if the unit map $l \rightarrow Z(\Lambda)$ is an isomorphism. We note that for the remainder of this paper we need only assume $\mathbf{V}$ is finitely bicomplete.

Let $\operatorname{Mon}(\mathbf{V})$ be the monoidal category with objects the monoids of $\mathbf{V}$ and maps the monoid isomorphisms. Let $\mathbf{M}$ be the category of Morita contexts with tensor product $\square$ and unit $I^{\prime}=([I] \rightarrow[I] \otimes G \leftarrow[I])$ as defined in [7]. Since we will be interested only in Morita contexts $M=(\mathbf{C}, T)$ with C. a $\mathrm{V}$-category with two objects 0 and 1 , we will use the notation

$$
M=(\mathbf{C}(0,0), \mathbf{C}(1,0), \mathbf{C}(0,1), \mathbf{C}(1,1))
$$

to specify the Morita contexts. The notation for $M$ as a cospan then becomes

$$
([\dot{\mathbf{C}}(1,1)] \rightarrow \mathbf{C} \leftarrow[\mathbf{C}(0,0)])
$$

and $\Phi\left(M^{t}\right)=\mathbf{C}(1,0) \otimes C_{(1,1)^{-.}}$. Define $\Psi: \operatorname{Mon}(\mathbf{V}) \rightarrow \mathbf{M}$ by $\Psi(\Lambda)=\left(\mathbf{C}_{\boldsymbol{\Lambda}}, T_{\boldsymbol{\Lambda}}\right)$ where $C_{\Lambda}$ is the full subcategory of $V^{\Lambda^{e}}$ with objects $\Lambda$ and $\Lambda^{e}$, and 
$T_{\Lambda}$ is defined by $T(\Lambda)=0$ and $T\left(\Lambda^{\mathrm{e}}\right)=1$. We have

$$
\Psi(\Lambda)=\left(Z(\Lambda), \Lambda, \mathrm{V}^{\Lambda^{\mathrm{e}}}\left(\Lambda, \Lambda^{\mathrm{e}}\right),\left(\Lambda^{\mathrm{e}}\right)^{\mathrm{o}}\right) \text {. }
$$

We note that $\Psi$ is a map of (symmetric) monoidal categories, i.e., we have natural maps

$$
\psi_{\Lambda_{1} \Lambda_{2}}: \Psi\left(\Lambda_{1}\right) \square \Psi\left(\Lambda_{2}\right) \rightarrow \Psi\left(\Lambda_{1} \otimes \Lambda_{2}\right)
$$

and a map $\psi_{0}: I^{\prime} \rightarrow \Psi(I)$ satisfying diagrams MF $1-\mathrm{MF} 4$ in $\left[5, \mathrm{pp}_{\mathrm{e}} 473\right.$, 513]. In this case $\psi_{0}$ is the identity and if $\Lambda_{i}$ is a $\mathbf{V}$-atom in $\mathbf{V} \boldsymbol{\Lambda}_{i}^{e}$ for $i=1$, 2, i.e., $\mathbf{V} \Lambda_{i}^{e}\left(\Lambda_{i},-\right)$ is $\mathbf{V}$-cocontinuous, then $\psi_{\Lambda_{1} \Lambda_{2}}$ is an isomorphism, i.e., $\Psi$ restricted to the category of such monoids is strict.

We call a monoid $\Lambda$ an Azumaya monoid if $\Lambda$ is central, $\Lambda$ is a $V$-atom in $V^{\Lambda^{e}}$ and the functor [I] $\rightarrow V^{\Lambda^{e}}$ induced by $\Lambda$ is $\mathbf{V}$-dense [6, after (1.17)]. In general if $\Omega_{0}$ and $\Omega_{1}$ are monoids and $B$ is a left $\Omega_{0}$, right $\Omega_{1}$ bimodule, i.e., $B$ induces a functor $\left[\Omega_{1}\right]^{\circ} \otimes\left[\Omega_{0}\right] \rightarrow \mathbf{V}$, then $B$ is said to be $\Omega_{0}-\Omega_{1}$ prodense if $B$ is a $\mathbf{V}$-atom in $\mathbf{V}^{\Omega_{0}}$ and the functor $B^{\#}:\left[\Omega_{1}\right]^{\circ} \rightarrow \mathbf{V}_{0}$ induced by $B$ is $\mathbf{V}$-dense and $\mathbf{V}$-full and faithful. This is equivalent to $B$ being a $\Omega_{0}-\Omega_{1}$-progenerator as defined in [3] if $\mathbf{V}$ is a category of left modules over a commutative ring. Thus $\Lambda$ is an Azumaya monoid if and only if $\Lambda$ is $\Lambda^{e}$-I-prodense.

Lemma 2. If $B$ is a left $\Omega_{0}$, right $\Omega_{1}$-bimodule the following statements are equivalent:

(a) $B$ is $\Omega_{0}-\Omega_{1}$-prodense.

(b) There is a Morita equivalence $M=\left(\Omega_{0}, B, D, \Omega_{1}\right)$.

(c) $B$ is $\Omega_{1}^{\circ}-\Omega_{0}^{\circ}$-prodense.

(d) $\mathbf{V}^{\Omega_{0}\left(B, \Omega_{0}\right)}$ is $\Omega_{0}^{\circ}-\Omega_{1}^{\circ}$-prodense and $\mathbf{V}^{\Omega_{0}}(B,-): \mathbf{V}^{\Omega_{0}} \rightarrow \mathbf{V}^{\Omega_{1}}$ is V-cocontinuous.

Proof. (a) $\Longrightarrow$ (b) since $B^{\#}$ is full and faithful, $\Omega_{1}=\mathbf{V}^{\Omega_{0}}(B, B)$, and we get the Morita context in (b) by taking $D=\mathbf{V}^{\mathbf{\Omega}_{0}}\left(B, \Omega_{0}\right)$ and using the composition maps in $\mathbf{V}^{\boldsymbol{\Omega}_{0}}$. Since $B$ is a $\mathbf{V}$-atom in $\mathbf{V}^{\boldsymbol{\Omega}_{0}}$ we have an isomorphism

$$
\mathbf{V}^{\Omega_{0}}\left(B, \Omega_{0}\right) \otimes_{\Omega_{0}} B \rightarrow \mathbf{V}^{\Omega_{0}}(B, B)=\Omega_{1}
$$

which implies $M$ has a right $*$-inverse. The density of $B^{\#}$ gives us that $M$ is a Morita equivalence by $[7$, Theorem 4]. (b) $\Rightarrow$ (a) follows from $[7$, Theorem 4]. (c) $\Leftrightarrow$ (b) follows from (a) $\Leftrightarrow$ (b) by observing that $M$ is a Morita equivalence if and only if $\left(M^{\circ}\right)^{t}=\left(\Omega_{1}^{\circ}, B, D, \Omega_{0}^{\circ}\right)$ is one. (b) $\Rightarrow$ (d) follows 
from (b) $\Rightarrow$ (a) applied to $M^{\circ}=\left(\Omega_{0}^{\circ}, D, B, \Omega_{1}^{\circ}\right)$, since $D \simeq \mathbf{V}^{\Omega_{0}}\left(B, \Omega_{0}\right)$ by [7, Theorem 4 (iii)] applied to $M$. (d) $\Rightarrow$ (a) follows from (a) $\Rightarrow$ (d) applied to $\mathbf{V}^{\Omega_{0}}\left(B, \Omega_{0}\right)$ if we show $B \simeq \mathbf{V}_{0}^{\Omega_{0}^{o}}\left(\mathbf{V}^{\Omega_{0}}\left(B, \Omega_{0}\right), \Omega_{0}^{o}\right)$, This isomorphism follows from the fact that

$$
\mathbf{V}^{\Omega_{0}}(B,-) \quad \text { and } \quad \mathbf{V}^{\boldsymbol{\Omega}_{0}}\left(\mathbf{V}^{\mathbf{\Omega}_{0}^{\circ}}\left(\mathbf{V}^{\boldsymbol{\Omega}_{0}}\left(B, \Omega_{0}\right), \Omega_{0}^{\circ}\right),-\right)
$$

are cocontinuous.

Let $I(\mathbf{V})$ be the monoidal subcategory of $\mathbf{V}$ with the same objects as $\mathbf{V}$ but with maps the isomorphisms of $\mathbf{V}$. Define the map of monoidal categories $\theta: I(\mathbf{V}) \rightarrow \mathbf{M}$ by $\theta(X)=\left(\mathbf{D}_{X}, T_{X}\right)$, where $\mathbf{D}_{X}$ is the full subcategory of $\mathbf{V}$ with objects $X$ and $I$ and $T_{X}$ is defined by $T_{X}(X)=0$ and $T_{X}(I)=$ 1. When $\theta$ is restricted to the $\mathbf{V}$-atoms of $\mathbf{V}$ it is a strict map. Note that $\theta(X)=\left(\mathbf{V}(X, X), X, X^{*}, I\right)$ where $X^{*}=\mathbf{V}(X, I)$.

Theorem 3. For any object $X$ of $\mathbf{V}$ the following statements are equivalent:

(a) The functor $[\mathbf{V}(X, X)] \rightarrow \mathbf{V}$ induced by $X$ is $\mathbf{V}$-dense and $X$ is a V-atom.

(b) $X$ is $\mathbf{V}(X, X)$-I-prodense.

(c) $\theta(X)$ is a Morita equivalence.

(c') $X \otimes: \mathbf{V} \rightarrow \mathrm{V}^{\mathrm{V}(X, X)}$ is an equivalence of categories.

(d) $\theta\left(X^{*}\right)$ is a Morita equivalence, $\mathrm{V}(X, X) \simeq \mathrm{V}\left(X^{*}, X^{*}\right)^{\circ}$, and $X$ is a V-atom.

Proof. Since (a) is equivalent to the statement that $X$ is $1-\mathbf{V}(X, X)^{\circ}$ prodense, Lemma 2 implies the equivalence of (a), (b), (c) and the statement that $X^{*}$ is $I^{\circ}-\mathbf{V}(X, X)^{\circ}$-prodense and $X$ is a $\mathbf{V}$-atom in $\mathrm{V}$. Note that if $X^{*}$ is $I^{\circ} \cdot \mathbf{V}(X, X)^{\circ}$-prodense, then $\left(X^{*}\right)^{\#}:[\mathbf{V}(X, X)]^{\circ} \rightarrow \mathbf{V}$ is full and faithful and we get $\mathbf{V}(X, X) \simeq \mathbf{V}\left(X^{*}, X^{*}\right)^{\circ}$. The equivalence of (c) and (c') follows from [7, Corollary 5] applied to $\theta(X)^{t}$, since $\Phi\left(\theta(X)^{t}\right)=X \otimes \rightarrow$

Theorem 4. For a monoid $\Lambda$ the following statements are equivalent:

(a) $\Lambda$ is an Azumaya monoid.

(b) $\Psi(\Lambda)$ is a Morita equivalence and $\Lambda$ is central.

(b') $\Lambda \otimes: \mathbf{V} \rightarrow \mathbf{V}^{\Lambda^{\mathrm{e}}}$ is an equivalence of categories.

(c) $\Lambda$ is a $\mathbf{V}$-atom in $\mathbf{V}$, the functor $\left[\Lambda^{\mathrm{e}}\right] \rightarrow \mathbf{V}$ induced by $\Lambda$ is dense and the canonical map $\Lambda^{\mathrm{e}} \rightarrow \mathrm{V}(\Lambda, \Lambda)$ is an isomorphism.

Proof. The equivalence of (b) and ( $\left.b^{\prime}\right)$ follows from [7, Corollary 5] since $\Phi\left(\Psi(\Lambda)^{t}\right)=\Lambda \otimes-$. The rest of the theorem follows from Lemma 2 . 
Theorem 5. (i) If $\Lambda_{1}$ and $\Lambda_{2}$ are Azumaya monoids then so is $\Lambda_{1} \otimes \Lambda_{2}$.

(ii) If $X_{i}$ is $\mathrm{V}\left(X_{i}, X_{i}\right)$-I-prodense, $i=1,2$, then $X_{1} \otimes X_{2}$ is $\mathbf{V}\left(X_{1} \otimes X_{2}, X_{1} \otimes X_{2}\right)$-I-prodense, $\mathbf{V}\left(X_{1}, X_{1}\right)$ is an Azumaya monoid, and $X_{1} \otimes X_{1}^{*} \simeq \mathrm{V}\left(X_{1}, x_{1}\right)$.

Proof. If $\Lambda_{1}$ and $\Lambda_{2}$ are Azumaya then $\Psi\left(\Lambda_{1} \otimes \Lambda_{2}\right) \simeq \Psi\left(\Lambda_{1}\right) \square \Psi\left(\Lambda_{2}\right)$. Thus, by Theorem 4 and [7, Theorem 3] $\Lambda_{1} \otimes \Lambda_{2}$ is Azumaya. If $X_{i}$ is $\mathbf{V}\left(X_{i}, X_{i}\right)$-I-prodense, then $X_{i}$ is a $\mathbf{V}$-atom, $i=1,2$, and $\theta\left(X_{1} \otimes X_{2}\right) \simeq$ $\theta\left(X_{1}\right)\left[\theta\left(X_{2}\right)\right.$. Thus by Theorem 3 and [7, Theorem 3] $X_{1} \otimes X_{2}$ is $\mathbf{V}\left(X_{1} \otimes X_{2}, X_{1} \otimes X_{2}\right)-I$-prodense. Since $X_{1}^{*}$ is $\mathbf{V}\left(X_{1}^{*}, X_{1}^{*}\right)$-I-prodense, we obtain

$$
\begin{gathered}
\theta\left(x_{1} \otimes x_{1}^{*}\right) \simeq \theta\left(x_{1}\right) \square \theta\left(x_{1}^{*}\right), \quad x_{1} \otimes x_{1}^{*} \simeq \mathbf{V}\left(x_{1}, x_{1}\right), \\
\mathbf{V}\left(x_{1}, x_{1}\right)^{\circ} \simeq \mathbf{V}\left(x_{1}^{*}, x_{1}^{*}\right) .
\end{gathered}
$$

This implies $\mathbf{V}\left(X_{1}, X_{1}\right)$ is $\mathbf{V}\left(X_{1}, X_{1}\right)^{\mathrm{e}}$-I-prodense and thus an Azumaya monoid.

We let $A(\mathbf{V})$ be the class of Azumaya monoids in $\mathbf{V}$ and $A_{0}(\mathbf{V})$ be the class of monoids $\mathbf{V}(B, B)$ in $\mathbf{V}$ where $B$ is $\mathbf{V}(B, B)-I$-prodense. By Theorem $5, A(\mathbf{V})$ and $A_{0}(\mathbf{V})$ are closed under $\otimes$, and $A_{0}(\mathbf{V})$ is a subclass of $A(\mathbf{V})$. Define an equivalence relation on $A(\mathbf{V})$ by $\Lambda_{1} \sim \Lambda_{2}$ if $\mathbf{V}^{\Lambda_{1}} \simeq$ $\mathbf{V}^{\Lambda_{2}}$. Let the Brauer group of $\mathbf{V}, B(\mathbf{V})$, be the equivalence classes under this equivalence relation. Theorems 3,4 and 5 imply that $B(V)$ is a group (here we allow groups to have a class of objects) with identity element $\operatorname{cl}(I)=\operatorname{cl}(\Omega)$ for all $\Omega$ in $A_{0}(\mathbf{V})$, multiplication defined by $\operatorname{cl}\left(\Lambda_{1}\right) \cdot \operatorname{cl}\left(\Lambda_{2}\right)=$ $\operatorname{cl}\left(\Lambda_{1} \otimes \Lambda_{2}\right)$ and inverse $\operatorname{cl}(\Lambda)^{-1}=\operatorname{cl}\left(\Lambda^{\circ}\right)$. We note that if $\mathrm{V}$ is cartesian closed then the unit object $I$ is the only $\mathrm{V}$-atom; hence $B(\mathrm{~V})$ is trivial by Theorem 4.

We now consider maps of monoidal categories which induce maps of Brauer groups. First we need an alternate description of the equivalence relation defined above.

Proposition 6. For $\Lambda_{1}$ and $\Lambda_{2}$ Azumaya monoids, $\Lambda_{1} \sim \Lambda_{2}$ if and only if there exist $\Omega_{1}$ and $\Omega_{2}$ in $A_{0}(\mathbf{V})$ such that $\Lambda_{1} \otimes \Omega_{1} \simeq \Lambda_{2} \otimes \Omega_{2}$.

Proof. The necessity is clear since $\mathbf{V}^{\Omega} \simeq \mathbf{V}$ for every $\Omega$ in $A_{0}(\mathbf{V})$ by Theorem 3. For the sufficiency suppose $V^{\Lambda_{1}} \simeq V^{\Lambda_{2}}$. Then we have $V \simeq V^{\Lambda_{1} \otimes \Lambda_{1}^{\circ}} \simeq V^{\Lambda_{2} \otimes \Lambda_{1}^{\circ}}$, which implies by [7, Corollary 5] that there is a Morita 
equivalence $\left(I, B, D, \Lambda_{2} \otimes \Lambda_{1}^{\circ}\right)$ with $B$ and $D$ in $\mathbf{V}$, i.e., by Lemma $2, B$ is $l-\Lambda_{2} \otimes \Lambda_{1}^{o}$-prodense. This implies $\Lambda_{2}^{\circ} \otimes \Lambda_{1} \simeq \mathbf{V}(B, B)$ and $\mathbf{V}(B, B)$ is in $A_{0}(\mathbf{V})$. Tensoring with $\Lambda_{2}$ gives the desired result since $\Lambda_{2} \otimes \Lambda_{2}^{\circ} \simeq$ $\mathrm{V}\left(\Lambda_{2}, \Lambda_{2}\right)$ is in $A_{0}(\mathbf{V})$.

Theorem 7. Let $\mathbf{V}$ and $\mathbf{V}^{\prime}$ be bicomplete closed categories and $T$ : $\mathbf{V} \rightarrow \mathbf{V}^{\prime}$ be a strict map of monoidal categories such that

(i) $T$ preserves atoms and coequalizers;

(ii) There exists an isomorphism $K_{B}: T(\mathbf{V}(B, I)) \rightarrow \mathbf{V}^{\prime}(T B, T I)$, for every atom $B$ in $\mathbf{V}$, which is natural in $B$.

Then $T$ induces a group bomomorphism $B(T): B(\mathbf{V}) \rightarrow B\left(\mathbf{V}^{\prime}\right)$.

Proof. Since $T$ is strict we need only show $T$ maps $A(\mathbf{V})$ into $A\left(\mathbf{V}^{\prime}\right)$ and $A_{0}(\mathbf{V})$ into $A_{0}\left(\mathbf{V}^{\prime}\right)$. But $T$ is strict implies $T\left(\Lambda^{\mathrm{e}}\right) \simeq T(\Lambda)^{\mathrm{e}}$. For $B$ a $\mathbf{V}$-atom in $\mathbf{V}$, the isomorphism $K_{B}$ induces natural isomorphisms

$$
K_{B, A}: T(\mathbf{V}(B, A)) \rightarrow \mathbf{V}^{\prime}(T B, T A)
$$

for every $A$ in $\mathbf{V}$ by the observation that for a $\mathbf{V}$-atom $B, \mathbf{V}(B, I) \otimes A \simeq$ $\mathbf{V}(B, A)$. Thus if $\Lambda$ is Azumaya $T(\Lambda)^{\mathrm{e}} \simeq \mathbf{V}(T \Lambda, T \Lambda)$. If $B$ is $\mathbf{V}(B, B)-I$ prodense then $B$ is an atom and hence $T B$ is one. To show $T B$ is $V^{\prime}(T B, T B)-I^{\prime}$-prodense, it suffices to show that the map

$$
f_{T B}: \mathrm{V}^{\prime}(T B, T I) \otimes_{\mathrm{V}(T B, T B)} T B \rightarrow I^{\prime}
$$

is an isomorphism. This follows from the facts that the corresponding map for $B$ is an isomorphism and

$$
\mathrm{V}^{\prime}(T B, T I) \otimes_{\mathrm{V}^{\prime}(T B, T B)}^{\prime} T B \simeq T\left(\mathrm{~V}(B, I) \otimes_{\mathrm{V}(B, B)} B\right)
$$

which holds since $T$ preserves coequalizers and is strict and we have the isomorphisms $K_{B I}$ and $K_{B B}$. Thus $T$ maps $A_{0}(\mathbf{V})$ into $A_{0}\left(\mathbf{V}^{\prime}\right)$ and by using the Theorem 4 (c) characterization of Azumaya monoids we see $T$ maps $A(\mathbf{V})$ into $A\left(\mathbf{V}^{\prime}\right)$.

Corollary 8. If $R$ and $S$ are commutative monoids in $\mathbf{V}$ and $t: R \rightarrow S$ is a map of monoids, then there is a canonical group homomorphism $B(t)$ : $B\left(\mathbf{V}^{R}\right) \rightarrow B\left(\mathbf{V}^{S}\right)$ which sends $\operatorname{cl}(\Lambda)$ to $\operatorname{cl}\left(S \otimes_{R} \Lambda\right)$.

Proof. We take $T=S \otimes_{R^{-}}: \mathbf{V}^{R} \rightarrow \mathbf{V}^{S}$, the left adjoint of $\mathbf{V}^{t}: \mathbf{V}^{S} \rightarrow$ $V^{R}$, and observe that $T$ satisfies the properties of Theorem 7 .

Thus, $B$ induces a functor from the category of commutative monoids in $\mathbf{V}$ to the category of Abelian groups. 


\section{REFERENCES}

1. B. Auslander, The Brauer group of a ringed space, J. Algebra 4 (1966), 220273. MR $33 \# 7362$.

2. M. Auslander and O. Goldman, The Brauer group of a commutative ring, Trans. Amer. Math. Soc. 97 (1960), 367-409. MR 22 \#12130.

3. H. Bass, The Morita theorems, University of Oregon (mimeographed notes).

4. - Lectures on topics in algebraic K-theory, Tata Institute of Fundamental Research Lectures on Math.,.no. 41, Tata Institute of Fundamental Research, Bombay, India, 1967. MR 43 \# 4885.

5. S. Eilenberg and G. M. Kelly, Closed categories, Proc. Conf. Categorical Algebra (La Jolla, Calif., 1965), Springer, New York, 1966, pp. 421-562. MR 37 \# 1432.

6. J. Fisher-Palmquist and D. C. Newell, Triples on functor categories, J. Algebra 25 (1973), 226-258. MR 48 \# 2218.

7. J. Fisher-Palmquist and P. H. Palmquist, Morita contexts of enriched categories, Proc. Amer. Math. Soc. 50 (1975), 55-60.

8. S. Mac Lane, Categories for the working mathematician, Springer-Verlag, New York and Berlin, 1971.

DEP ARTMENT OF MATHEMATICS, UNIVERSITY OF CALIFORNIA, IRVINE, CALIFORNIA 92664 